\title{
UNA CLASIFICACIÓN DE LA EDUCACIÓN EMPRESARIAL: DIFERENCIAS ENTRE LOS ESTADOS UNIDOS Y EUROPA
}

\section{A CLASSIFICATION OF BUSINESS EDUCATION: DIFFERENCES BETWEEN THE UNITED STATES AND EUROPE}

\author{
Francisco Liñán Alcalde ${ }^{1}$ \\ ${ }^{1}$ Departamento Economía Aplicada I, Universidad de Sevilla, flinan@us.es
}

Recebido para publicação em: 15/10/2004

Aceito para publicação em: 05/03/2005

\section{RESUMEN}

La educación empresarial ha adquirido un gran desarrollo en las últimas décadas. Este auge ha sido simultáneo al de la figura del empresario, al que se le concede cada vez un mayor papel en los procesos de cambio estructural de las economías. Cada vez más, se reconoce la necesidad de más y mejores empresarios para el dinamismo de las economías. En este sentido, la educación empresarial parece una de las estrategias más adecuadas para la promoción empresarial. Sin embargo, al igual que con la propia definición de empresario, no existe unanimidad en cuanto al concepto u objetivos de la educación empresarial. Así, un programa formativo que persiga la aparición de empresarios puede considerarse una iniciativa de educación empresarial. No obstante, desde diferentes ámbitos se amplía esa visión en una dirección o en otra. En particular, la diferente visión predominante en los Estados Unidos y Europa sobre la actividad empresarial en general, y sobre la educación empresarial en particular, han supuesto un desarrollo muy diferente de ésta última. El objetivo principal de este trabajo es analizar las diferencias sobre educación empresarial existentes a cada lado del Atlántico, y las consecuencias derivadas de ello. Esas diferencias permitirían explicar la distinta evolución seguida por la educación empresarial en ambas zonas, así como sus distintos objetivos. En el caso de Europa, la educación empresarial se ha difundido más recientemente, y sus objetivos son abiertamente más amplios que los que se persiguen en Estados Unidos.

Palabras clave: Educación empresarial; promoción empresarial; Estados Unidos y Europa.

\section{Introducción}

En la actualidad existen grandes retos a los que se tienen que enfrentar las diferentes economías, tanto las nacionales como las regionales. Las nuevas tecnologías de la información y la comunicación, el proceso de globalización con su secuela de creciente competencia, la reforma del papel del Estado, todas ellas pueden ser amenazas muy serias para las regiones y países, especialmente para aquellos económicamente menos desarrollados. Frente a esta nueva situación se intentan buscar soluciones que, en muchos casos, han pasado por proclamar la gran relevancia de la actividad empresarial y, en consecuencia, de los empresarios y las pequeñas y medianas empresas. 
En este sentido, han comenzado a surgir una serie de iniciativas que pretenden promover la aparición de empresarios como instrumento para dinamizar las economías y promover la flexibilidad del tejido productivo y, de esta forma, el cambio estructural. Entre éstas, cabe citar la promoción del espíritu empresarial, la simplificación de los trámites para la creación de empresas, o las políticas que de una u otra forma tratan de compensar a las pequeñas empresas por su supuesta desventaja frente a las grandes (OCDE, 1999; COMISIÓN EUROPEA,2003).

Dentro de estas medidas, destaca en particular, por su gran crecimiento en los últimos años, lo que se ha dado en llamar la "educación empresarial". Sin embargo, no existe acuerdo entre los investigadores sobre la concepción ni sobre los objetivos que debe perseguir este tipo de formación. Por lo tanto, se dan en la práctica una gran cantidad de variedades diferentes. Así, las actividades formativas que incluyen en sus contenidos el análisis de la figura del empresario, o las que se centran en el proceso de creación y/o desarrollo de empresas podrían ser consideradas como educación empresarial.

A pesar de esa falta de consenso, pueden distinguirse dos visiones alternativas de la educación empresarial que se encuentran relativamente difundidas, respectivamente, en los Estados Unidos de América y en Europa. En el primer caso, es muy habitual la concentración de los esfuerzos formativos en los pasos concretos para la puesta en marcha de una empresa. Por el contrario, en el caso europeo, frecuentemente se persigue el desarrollo de personalidades innovadoras de una forma más general. En nuestra opinión, esa diferente concepción se puede explicar, en buena parte, por el distinto desarrollo que ha seguido este tipo de formación en ambas áreas. Del mismo modo, esa distinta concepción estaría condicionando la traslación a la práctica de la educación empresarial, y -en consecuencia- los resultados obtenidos.

El objetivo del presente trabajo es el de estudiar las diferencias tanto teóricas como prácticas existentes entre las visiones norteamericana y europea de la educación empresarial. Para ello, después de esta introducción, la segunda sección recoge brevemente la importancia actual del empresario y de las pequeñas y medianas empresas, lo cual permitiría justificar la utilidad de la educación empresarial. A continuación tratamos de clarificar el concepto de educación empresarial. Las secciones cuarta y quinta se dedican a precisar las visiones de la educación empresarial existentes en esas dos áreas de referencia. Por último, el sexto apartado recoge las conclusiones principales de este trabajo.

\section{2. importancia del empresario y de la educación empresarial}

La importancia concedida recientemente a la educación empresarial como instrumento de competitividad se justifica por el creciente papel que está asumiendo el agente empresarial. En este 
sentido, aunque está claro que el empresario y la pequeña empresa son dos conceptos diferentes, la afinidad entre los mismos es elevada (WENNEKERS; THURIK, 1999; STOREY, 1982). Por un lado, si bien en toda empresa existe al menos un empresario, en las pequeñas es normalmente mucho más fácil identificarlo, ya que éste es al mismo tiempo propietario, gestor, y promotor de la misma. Así, las nuevas empresas independientes (es decir, que no son filiales de otra empresa mayor) son necesariamente creadas por uno o varios empresarios individuales. En sentido contrario, aunque puede existir actividad empresarial que no se refleje en la creación y dirección de una pequeña empresa (GIBB, 1990; PLASCHKA; WELSCH, 1990), parece correcto afirmar que una de las formas más evidentes y usuales de poner en práctica una iniciativa empresarial es a través de la creación de una empresa.

Por ello, teniendo en cuenta esa estrecha relación entre empresario y pequeña empresa, creemos necesario dedicar unas líneas al análisis de la creciente importancia que han venido adquiriendo las pequeñas y medianas empresas en los procesos de ajuste y reestructuración de las economías. Esa mayor presencia, además, ha venido acompañada por una creciente atención por parte de los académicos al concepto de función empresarial, y a su papel en el desarrollo económico.

Respecto al primero de esos aspectos, numerosos autores han señalado la tendencia a la concentración empresarial predominante durante la mayor parte del siglo XX (STOREY, 1988; PIORE; SABEL, 1984). Por el contrario, a raíz del estallido de la crisis económica de los años setenta se detecta un importante cambio de tendencia. Comienza a ponerse de manifiesto una creciente presencia de pequeñas y medianas empresas en la economía, que cada vez cobran un mayor protagonismo en distintos aspectos como la creación de empleo, la producción, la innovación o la exportación. Así, por ejemplo, Acs y Audretsch (1990) señalan que las pequeñas empresas crearon más de la mitad del empleo en EE.UU. entre 1976 y 1986.

Por su parte, Schwalbach (1994), analiza cinco países europeos (Reino Unido, Alemania, Francia, Italia y Dinamarca) entre 1979 y 1986. Sus resultados indican que las pequeñas empresas aumentaron su participación en el número total de empresas a razón de un $0.35 \%$ anual. Es decir, cada vez hay más empresas pequeñas en relación a las de otros tamaños. Además, estas empresas también aumentaron su participación en las ventas a un ritmo del 1.33\% anual.

Otro aspecto importante en el que se destaca el papel de las pymes es en la innovación. Las pequeñas empresas no parecen encontrarse en situación de desventaja respecto a las grandes en relación a la innovación. Es más, el hecho de que el número relativo de patentes e innovaciones (por unidad monetaria invertida, por empleado, etc.) descienda con el tamaño de la empresa parece indicar que las pymes tienen de hecho una ventaja en la innovación (COHEN, 1992; KLEPPER, 1996). 
También en los países en desarrollo se está produciendo un aumento similar de la presencia de las pymes en el conjunto de la economía. Rondinelli y Kasarda (1992) señalan la gran presencia de pequeñas y medianas empresas y -sobre todo- de microempresas en estos países. La mayoría de ellas operan en el sector informal de la economía, proporcionando más de la mitad del empleo en las grandes ciudades, y entre el 70 y el $90 \%$ en las ciudades medianas.

Entre las explicaciones de ese resurgir de la pequeñas empresa, Acs; Audretsch (1990), sintetizan los factores explicativos más relevantes mencionados por los distintos autores. Según ellos, las seis causas fundamentales a tener en cuenta serían los siguientes:

- El desarrollo de nuevas tecnologías flexibles. Este sería el factor más decisivo, siguiendo a Piore; Sabel (1984), Storey (1988) o Loveman; Sengenberger (1991), especialmente por su efecto neutralizador de las tradicionales economías de escala.

- La creciente globalización de los mercados. La mayor competencia por parte de competidores extranjeros, junto con la volatilidad de los tipos de cambio, han supuesto que la flexibilidad productiva y organizativa sean cada vez más esenciales (STOREY, 1988).

- Cambios en la composición del trabajo. No sólo sería importante el mayor nivel educativo y menores exigencias salariales señaladas por Brock; Evans (1989). También habría que añadir la incorporación de la mujer al mercado de trabajo, que ha llevado a un intenso crecimiento del autoempleo femenino.

- Diversificación de la demanda de los consumidores. Debida a los mayores niveles educativos y de renta, así como al mayor acceso a la información sobre costumbres y culturas del resto del mundo. Todo ello habría hecho despertar demandas cada vez más diversas, con lo que se crearían nichos de mercado para nuevas pequeñas empresas (STOREY, 1988; BROCK; EVANS, 1989).

- Desregulación en numerosos mercados. Esta simplificación legislativa habría contribuido a acelerar el crecimiento de las pymes. Sin embargo, puesto que no comenzó a darse hasta los años ochenta, no se hallaría en la raíz del fenómeno (STOREY, 1988; BROCK; EVANS, 1989).

- Un periodo de “destrucción creativa" schumpeteriana. La aceleración en el ritmo de introducción de innovaciones, así como el hecho de que una parte más que proporcional de las mismas es atribuible a las nuevas pequeñas empresas, según Kirchhoff (1990) o Brock; Evans (1989), sería una prueba de que estamos en una de esas etapas.

El interés de la clase política por el factor empresarial, por su parte, debe mucho al hecho de que las pequeñas empresas se hayan convertido en los más importantes creadores de empleo en una época de alto nivel de desempleo (BIRCH, 1979; ACS; AUDRETSCH, 1990). Sin embargo, no debemos conformarnos con ese papel. El empresario, fundamentalmente a través de la creación de nuevas pequeñas empresas y del desarrollo de las existentes, contribuye a elevar el grado de desarrollo de la economía en la que opera. Parece, por tanto, que existe una relación a nivel general 
entre grado de desarrollo y densidad empresarial (STOREY, 1982). De forma más específica, se puede afirmar que el surgimiento y desarrollo de nuevas pequeñas empresas independientes:

- Permite el rejuvenecimiento del tejido productivo. Kirchhoff (1990) enfatiza la importancia de la entrada de nuevas empresas para mantener el dinamismo del sistema. En el caso de la U.E., las nuevas empresas creadas cada año suponen en torno a un $12 \%$ del stock total de empresas existentes (COMISIÓN EUROPEA, 1998).

- Eleva la flexibilidad de ese tejido. En particular, la incorporación de tecnologías flexibles parece estar claramente relacionada con el descenso del tamaño medio de las empresas (ACS et al., 1990).

- Incrementa su grado de integración productiva. Las nuevas pequeñas empresas suelen establecerse por individuos de la misma región donde se instalan (MAILLAT, 1988), situándose la mayor parte de sus proveedores y clientes en su entorno local (STOREY, 1982; SANTOS, 2001).

Por todo ello, esas nuevas empresas independientes tendrán un efecto multiplicador mayor sobre el resto del tejido productivo local, creando riqueza y empleo de forma indirecta (FISHER, 1988). Del mismo modo, una superior presencia de pequeñas y medianas empresas independientes en una determinada rama productiva la dota de un mayor dinamismo económico, al tiempo que la hace menos dependiente de decisiones foráneas, contribuyendo a un tejido productivo más integrado y sólido (CARREE; THURIK, 1998). En la misma línea, Nickel (1996), analiza el impacto de la competencia en el nivel y el crecimiento de la productividad, encontrando que la mayor competencia (número de empresas en la industria) se asocia con mayores tasas de crecimiento de la productividad.

La existencia de un elevado ritmo en la creación de empresas, así como una alta presencia de pymes, serían por tanto factores de desarrollo económico importantes, por los motivos apuntados. Además, también habría que tener en cuenta el papel de esas empresas como "semilleros" de otras empresas (AYDALOT, 1988). Una parte de esas nuevas empresas creadas crecen hasta convertirse en medianas o grandes unidades (STOREY, 1982).

\section{Problemas conceptuales}

De la misma forma que el interés por la empresarialidad ha venido creciendo tanto en los círculos académicos como en los políticos desde la crisis de los años setenta, también la educación empresarial ha experimentado un rápido auge en todo el mundo. Las teorías actuales sobre el desarrollo económico y el ajuste estructural de las economías incluyen la promoción empresarial como uno de los instrumentos imprescindibles. En este ámbito, la educación empresarial podría 
señalarse como una estrategia potencialmente muy efectiva (LIÑÁN, 2001). Para ello, sería necesario establecer una cierta delimitación de los diferentes tipos de educación empresarial existentes.

Al igual que ha ocurrido en relación a la empresarialidad como disciplina científica, la educación empresarial ha logrado un reconocimiento y crecimiento muy importantes en las últimas décadas (BLACKMAN y THOMPSON, 1987; SBA, 2000; Comisión Europea, 1999). Sin embargo, también en este campo, debido a la evidente y estrecha relación entre los dos disciplinas, nos encontramos con un problema relativo a la conceptualización de la misma. En efecto, la educación empresarial se desarrolla a partir del estudio de la actividad empresarial (SEXTON y BOWMAN, 1984), por lo que la falta de consenso en cuanto al concepto de empresario acarrea necesariamente la imposibilidad de desarrollar una definición generalmente aceptada sobre qué debe entenderse como educación empresarial.

En este sentido, existen numerosos intentos de conceptuar esta forma educativa. Los más simples la identifican con la formación para la creación de empresas. Así hacen, por ejemplo, McIntyre y Roche (1999: p. 33) al afirmar que se trata de un «proceso que proporciona a los individuos los conceptos y capacidades para reconocer oportunidades que otros han pasado por alto, y la intuición y la autoestima para actuar donde otros han dudado. Incluye instrucción sobre reconocimiento de oportunidades, gestión de los recursos en situaciones de riesgo, e iniciación de una iniciativa empresarial. También incluye instrucción sobre procesos de gestión empresarial tales como planificación, capitalización, marketing y análisis de flujos de caja».

En cambio, las concepciones más amplias abarcan una gran cantidad de objetivos y de fases diferentes que suelen incluir la formación a lo largo de todo el sistema educativo. A modo de ejemplo, la visión del Consortium for Entrepreneurship Education afirma que la educación empresarial debería considerarse como un modelo de aprendizaje permanente a lo largo de toda la vida. Entre sus características más importantes destaca el hecho de que cada una de las cinco etapas en que se divide son formalmente independiente, y se desarrollan sin necesidad de una coordinación estrecha entre ellas. No obstante, la conjunción de las mismas puede tener un efecto muy notable sobre el nivel de espíritu empresarial de una sociedad, sobre las tasas de creación de empresas, y también sobre su supervivencia y el dinamismo posterior de las mismas. Un enfoque parecido, aunque no tan amplio, es el que defiende la Comisión Europea (2002).

En los países en desarrollo, por su parte, también existe una considerable presencia de este tipo de iniciativas. Allí, como cabía esperar, la preocupación principal es el desarrollo económico. Estas experiencias, frecuentemente denominadas Programas de Desarrollo Empresarial, han adquirido un notable grado de difusión, debido a su más que razonable nivel de éxito (LOUCKS, 1988). En estos casos, aunque no suele existir una definición explícita de la educación empresarial, 
el objetivo casi siempre consiste en tratar de promover la creación efectiva de empresas. Sin embargo, se trata normalmente de programas con un contenido muy básico, que muchas veces incluyen la enseñanza de un oficio al mismo tiempo que promueven es establecimiento como artesanos independientes de los participantes.

En nuestra opinión, una concepción suficientemente amplia para permitir abarcar a las anteriores sería a siguiente (LIÑÁN, 2004: p. 163): «el conjunto de todas las actividades de formación -reglada o no reglada- que intentan desarrollan en los participantes la intención de ser empresario, o algunos de los elementos que influyen sobre esa intención, como el conocimiento empresarial, la deseabilidad de la actividad empresarial, o su factibilidad. En su sentido más general, abarcaría el desarrollo de los conocimientos, capacidades, actitudes y cualidades personales identificados con la empresarialidad. Además, concretamente para los colectivos en edad laboral, la educación empresarial pretende dotar a los participantes de la capacidad y el deseo suficientes para la efectiva creación de empresas, y para la dinamización posterior de las mismas».

Esta definición presenta una serie de rasgos característicos que consideramos que la hacen útil como marco de referencia para el análisis y la clasificación de las distintas iniciativas existentes. En primer lugar, pretende abarcar todas las actividades de formación, no sólo las desarrolladas dentro del sistema educativo reglado. En segundo lugar, sus objetivos no incluyen sólo la difusión de una cultura empresarial y la creación de empresas, sino que además pretende elevar el grado de dinamismo de los nuevos empresarios y de las empresas en funcionamiento -o calidad empresarial-.

Además, esta definición permite establecer un límite claro entre las actividades de educación empresarial, de un lado, y la formación en aspectos de gestión -como por ejemplo los estudios de administración y dirección de empresas, o cursos específicos sobre distintas vertientes de la gestión de empresas-, del otro. Así, estos últimos no se preocuparían de los rasgos, habilidades, actitudes o intenciones del sujeto, sino solamente de los conocimientos técnicos necesarios para la dirección de empresas. Del mismo modo, la formación convencional en gestión no se ocuparía del proceso de creación o de dinamización de un proyecto empresarial independiente, sino solamente de la administración de empresas en funcionamiento.

Al tratarse de una definición muy amplia, caben en ella gran variedad de iniciativas, por lo que se hace necesario establecer criterios para clasificarlas. De este modo, McMullan y Gillin (1998), basándose en el esquema teórico desarrollado previamente por McMullan y Long (1987), seleccionan seis elementos que consideran definitorios de un proyecto de educación empresarial: a) los objetivos que se persiguen; b) el profesorado encargado de impartirlo; c) los estudiantes participantes; d) el contenido de los cursos; e) los métodos de enseñanza; y f) las actividades específicas de apoyo para que los participantes pongan efectivamente en marcha su proyecto. 
Por razones de espacio, en este trabajo sólo comentamos el primero de ellos pues, como señala Brockhaus (1992), se trata de una cuestión fundamental, a la cual deberán quedar sometidos los restantes elementos tales como participantes, contenidos, pedagogía, o evaluación. Según McMullan y Long (1987), el objetivo último que debe perseguir la educación empresarial es la creación de empresas, y su éxito por tanto se debería medir por su impacto socioeconómico. No obstante, son varios los autores que no comparten esa concepción. Así, por ejemplo, Plaschka y Welsch (1990) insisten en que la creación de una nueva empresa no es la única opción que existe dentro de la carrera empresarial.

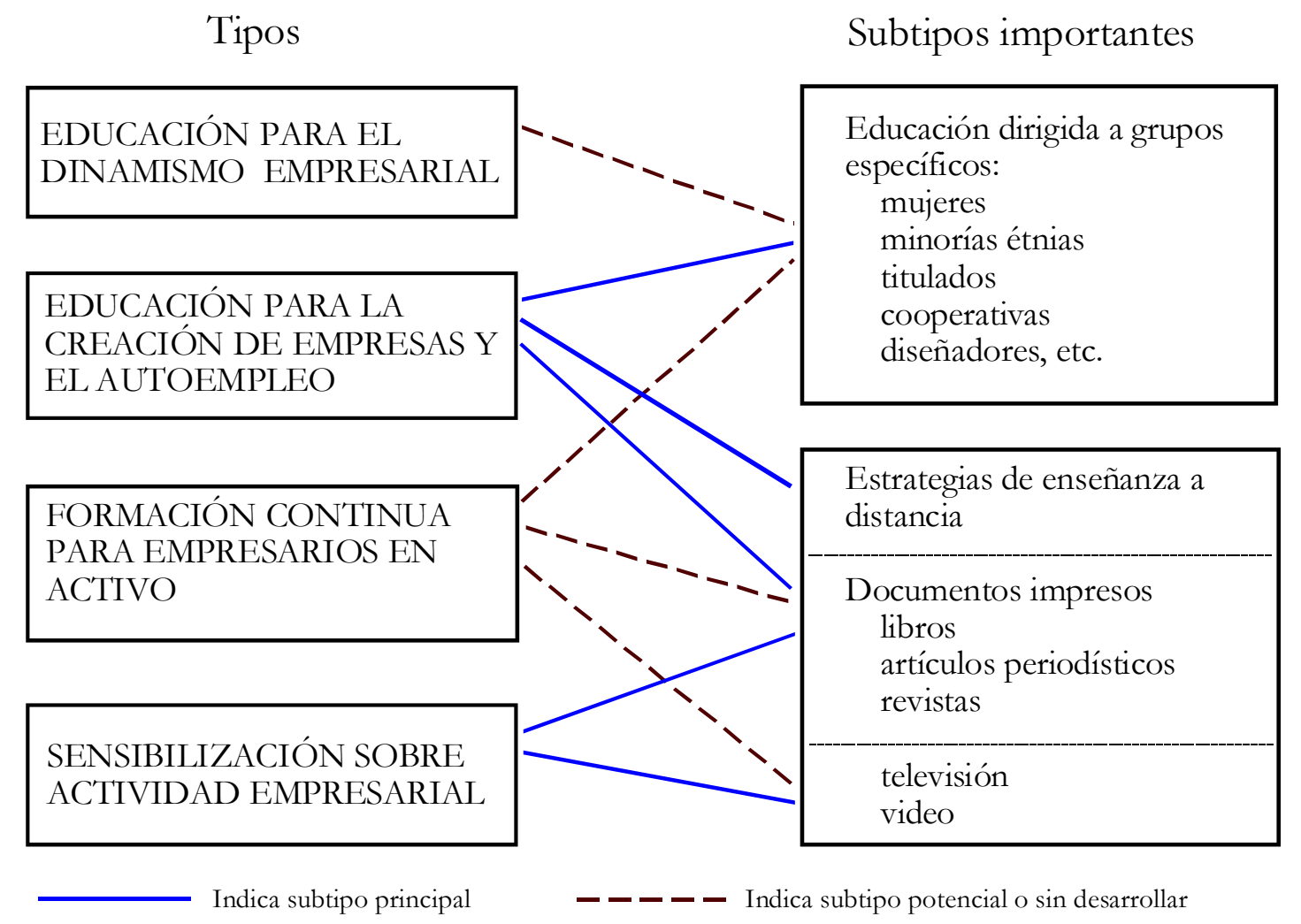

Figura 1 - Tipos de Educación Empresarial*

Precisamente en este sentido, Curran y Stanworth (1989) tratan de delimitar los principales tipos de objetivos que pueden perseguirse en la formación relacionada con la pequeña empresa y la actividad empresarial. En la Figura 1 se recoge la clasificación de esos autores, la cual ha sido asumida y defendida posteriormente por Garavan y O’Cinneide (1994) o Liñán (2004). A continuación pasamos a describir esas cuatro categorías siguiendo en buena medida las aportaciones de esos autores, pero introduciendo los matices necesarios para adaptarlas a nuestra concepción.

\footnotetext{
${ }^{*}$ CURRAN, J.; STANWORTH, J. (1989), Education and training for enterprise: some problems of classification, evaluation, policy and research, International Small Business Journal, v. 7, n. 2, p. 11.
} 
- Sensibilización hacia la actividad empresarial. Su finalidad es incrementar el número de personas que tienen un suficiente conocimiento sobre las pequeñas empresas y el autoempleo, de forma que consideren esa alternativa como una opción racional y viable. De esta forma, no persigue directamente la creación de empresarios. Ejemplo de este tipo de iniciativas son los cursos impartidos en las universidades. Se trata normalmente de cursos optativos dentro de las titulaciones de las áreas de economía e ingeniería, en las que los profesores no tratan de convertir a los estudiantes en empresarios, sino permitirles que en el futuro realicen su elección de carrera profesional con mayor perspectiva. Este tipo de cursos se adapta muy bien a las características de la enseñanza universitaria y, sobre todo, de la secundaria, por lo que su inclusión en esa etapa resulta muy conveniente (GARAVAN; O'CINNEIDE, 1994). De hecho, muchos de los cursos para la creación de empresas y el autoempleo -especialmente los de menor duración- estarían en realidad funcionando como programas de sensibilización (CURRAN; STANWORTH, 1989).

- Educación para la creación de empresas. Consiste en la preparación para ser propietarios de pequeños negocios convencionales, que es lo que son la gran mayoría de las nuevas empresas creadas. Se centra en los aspectos prácticos concretos relacionados con la creación de la empresa: cómo obtener financiación; regulaciones legales; fiscalidad; etc (CURRAN; STANWORTH, 1989). Los participantes en este tipo de cursos están, normalmente, muy motivados e incluso ilusionados con el proyecto, por lo que su receptividad hacia los contenidos de los mismos suele ser muy alta. Con frecuencia, el criterio de selección es el de tener ya una idea de negocio viable. Sin embargo, existen dificultades importantes que es necesario resolver para lograr un suficiente grado de efectividad de los programas. En concreto, Curran; Stanworth (1989) se refieren a tres tipos de obstáculos. En primer lugar, las percepciones que tienen los docentes y los alumnos sobre lo que debe contenerse en los cursos para la creación de empresas son muchas veces diferentes. Un segundo problema se refiere a la estructura de contenidos y duración que permite lograr la máxima efectividad de esos cursos. Por último, es preciso incorporar los aspectos necesarios para que el futuro empresario comprenda y asuma los distintos roles que conforman esa carrera profesional.

- Educación para el dinamismo empresarial. Es la encargada de promover unos comportamientos empresariales dinámicos y de calidad posteriores a la fase de creación de la empresa. Por tanto, su objetivo no es sólo elevar la intención de ser empresario, sino también la intención de desarrollar comportamientos dinamizadores cuando la empresa ya esté en marcha. Sin embargo, las formas convencionales de educación no permiten desarrollar la calidad empresarial, por lo que se precisaría utilizar modelos formativos alternativos que sí lo permitieran (GARAVAN; O'CINNEIDE, 1994). Esos autores reflexionan sobre aspectos pedagógicos de lo que sería una educación para el 
dinamismo empresarial, y analizan seis ejemplos europeos que se encuadran dentro de esta categoría.

- Formación para empresarios en activo. La última variedad de educación empresarial es la formación continua para pequeñas empresas. Se trata de una versión especializada de la educación de adultos o formación continua en general, diseñada para permitir mejorar y actualizar las habilidades de los empresarios en activo (WEINRAUCH, 1984). En particular, resulta complejo atraer a esos empresarios hacia este tipo de programas formativos, ya que tienden a considerar estas iniciativas demasiado generales para las necesidades concretas de su empresa. Una posible forma de superar esa dificultad podría encontrarse en la vinculación con las modalidades descritas anteriormente. En efecto, aquellos que han participado en algún programa de creación o de dinamización empresarial podrían ser más receptivos a la idea de una formación continua para la pequeña empresa.

Para concluir, los autores citados consideran que los cuatro posibles objetivos de la educación empresarial precisan de mucha más investigación que permita ampliar su base de conocimientos, perfeccionar sus técnicas de enseñanza, mejorar su efectividad y delimitar y avanzar hacia el logro de todo su potencial. En todo caso, coinciden en señalar la mayor trascendencia y relevancia de la educación para el dinamismo empresarial (GARAVAN; O'CINNEIDE, 1994; LIÑÁN, 2004).

\section{Visión de la educación empresarial en EE.UU.}

Dentro de los Estados Unidos, dado el alto grado de descentralización del sistema educativo, no existe un sistema nacional de educación empresarial, por lo que las prácticas y las concepciones varían significativamente de unas instituciones a otras, y de unos investigadores a otros. No obstante, parece predominar un enfoque mucho más concreto y pragmático en líneas generales. Según Gibb (1993: p. 29): «la educación empresarial en los Estados Unidos tiene claramente como su principal objetivo la creación de una conciencia, una comprensión y una motivación hacia la propiedad de pequeñas empresas independientes». Sin embargo, no todos los autores norteamericanos están de acuerdo con esa idea de propiedad independiente de una empresa. Para otros, lo importante es que se realice una actividad que genere valor añadido a la economía, y que los esfuerzos se vean recompensados mediante un ingreso, independientemente de la forma legal o institucional con la que se desarrolle esa actividad (TIMMONS, 1999). En cualquier caso, en ese país se entendería que la educación empresarial debe perseguir la efectiva realización por parte de los alumnos de esos comportamientos, lo cual se correspondería con lo que hemos denominado educación para la creación de empresas. 
De hecho, este parece ser un rasgo característico de la educación empresarial estadounidense desde sus inicios. Ya en 1947, Myles Mace estableció una asignatura optativa en el Master en Administración de Empresas de la Harvard Business School, denominada 'Gestión de nuevas empresas'. Su finalidad era la de facilitar la adaptación a la vida civil de los estudiantes que habían servido como soldados en la Segunda Guerra Mundial. Según Katz (2003), esa asignatura fue cursada por 188 de los 600 alumnos del master.

El hecho de que fuese precisamente la Universidad de Harvard la primera institución en ofrecer un curso de este tipo estaría relacionado con la presencia allí de Schumpeter. Así, es muy conocida la importancia que ese autor concedía al papel del empresario en la economía en general, y en el desarrollo económico en particular (SCHUMPETER, 1911). Él mismo fue cofundador del Research Center for Entrepreneurial History en esa Universidad en 1946. Posteriormente, en 1949 comenzó a publicarse también allí la primera revista dedicada al estudio del empresario: Explorations in Entrepreneurial History (KATZ, 2003).

A partir de ahí, el crecimiento en el número de instituciones de educación superior que ofrecen formación empresarial ha sido constante, si bien no es fácil determinar su ritmo. Algunos autores señalan la existencia de cuatro centros que ofrecían ese tipo de formación en 1968, que crecerían hasta 16 en 1970, acelerando su ritmo hasta alcanzar una cifra superior a las 400 instituciones en 1995 (VESPER; GARTNER, 1997). Sin embargo, en un estudio elaborado para la Small Business Administration, se cifra en 263 el número de instituciones que ofrecen educación empresarial en 1979, que crecerían hasta unos 1400 centros en 1998 (SOLOMON et al., 1994). A pesar de la significativa discrepancia que existe entre ambos estudios, se puede afirmar sin ninguna duda que el crecimiento ha sido muy importante.

Sin embargo, la educación empresarial en las universidades norteamericanas no sólo ha ido creciendo en número de centros, sino que esta tendencia se ha complementado con otra hacia la "profundización". En efecto, los cursos que comenzaron a ofrecerse como optativos en determinados masters y programas de postgrado, han ampliado su oferta en dos líneas. En primer lugar, se ha tendido a ofrecer combinaciones de cursos dentro de los programas de postgrado (concentrations), las cuales agrupan a un conjunto de cursos relacionados con el tema. En segundo lugar, se ha ido introduciendo en los niveles de grado como cursos sueltos al principio, y como agrupaciones de cursos posteriormente, que a su vez pueden ser áreas de especialización (minors) o auténticas especialidades (majors), que posibilitan la existencia de graduados en "entrepreneurship". Así, según Katz (2003), en 1968 el Babson College introdujo la primera área de especialización en empresarialidad a nivel de grado (minor). En 1971, la University of Southern California introdujo la primera combinación de cursos sobre empresarialidad a nivel de master. 
El movimiento hacia la creación de programas en educación empresarial (concentrations, minors o majors) se ha ido desarrollando a lo largo de la década de los setenta, aunque de forma más lenta. En este sentido, a la altura de 1979, tan sólo ocho centros ofrecían concentrations o majors en este campo, y eso incluyendo tanto los estudios de grado como los de postgrado (VESPER, 1982). Entre ellos destacaban, por la amplitud de su oferta formativa, Northeastern University, University of Southern California, y Wharton School, con entre ocho y nueve cursos diferentes cada una.

Este desarrollo tan rápido de la educación empresarial en los Estados Unidos no se debe sólo a la demanda por parte de los alumnos. Según Vesper (1982), la Administración Federal contribuyó grandemente mediante algunos de sus proyectos. Merece la pena destacar tres iniciativas especialmente. La primera, en la segunda mitad de los años sesenta, consistió en la generosa financiación otorgada por la National Science Foundation para la puesta en marcha de tres centros de innovación, que se crearon en las escuelas de ingeniería del Instituto Tecnológico de Massachusetts (MIT) y de Carnegie-Mellon, así como en la facultad de empresariales de Oregon. Estos centros coincidieron en apostar por la empresarialidad como un elemento central en el proceso de innovación, por lo que desarrollaron proyectos de educación empresarial que luego han servido de modelos a otras instituciones.

En segundo lugar, y más importante aún, estaría la actuación de la Administración para la Pequeña Empresa (SBA). Así, en 1972 este organismo lanzó un programa denominado Small Business Institute (SBI), consistente en la realización de trabajos de consultoría para las pequeñas empresas por parte de estudiantes universitarios de empresariales (SBA, 2002). Para ello, el SBI pagaba por cada trabajo de consultoría una cantidad a la facultad cuando el caso se concluía satisfactoriamente, sin ningún compromiso ni atadura en cuanto al destino de esa financiación. Por tanto, resultaba una iniciativa muy atractiva para las universidades.

De esta forma, este programa se difundió muy rápidamente a gran número de facultades de empresariales. El primer año de funcionamiento del SBI, éste se puso en marcha en una veintena de universidades, llegando hasta las 450 en 1995 (KATZ, 2003). Así, las facultades típicamente introdujeron un curso que consiste en la realización de esos trabajos de consultoría por parte de los alumnos. Sin embargo, para que los alumnos sepan adaptar sus conocimientos a la realidad de la pequeña empresa, es muy frecuente que se imparta como pre-requisito un curso sobre gestión de pequeñas empresas y, también con frecuencia, sobre creación de nuevas empresas. Por lo tanto, el programa SBI ha supuesto probablemente el mayor impulso a la educación empresarial en la década de los setenta, a pesar de que ese no era inicialmente su objetivo (KENT, 1990).

Un tercer aspecto a tener en cuenta es el referido a los Centros de Desarrollo Empresarial (Small Business Development Centers, SBDCs), que también son promovidos por la SBA a partir 
de 1977 como programa piloto, y desde 1980 como programa plenamente operativo. Estos centros pretenden ofrecer asesoría técnica y formación en todos los aspectos de la gestión de una pequeña empresa. Los centros se crean mediante acuerdo entre la SBA y uno o más socios estatales, que pueden ser públicos o privados, destacando como socios las universidades. En cualquier caso, lo normal es que en cada estado exista un sólo centro, pero con una serie de subsedes que se localizan prioritariamente en universidades, centros de formación, cámaras de comercio o agencias de desarrollo. Por lo tanto, aunque estos centros han promovido la formación empresarial más bien de forma marginal -a través de los programas de capacitación para empresarios-, sí han jugado un papel relevante en el aumento de la visibilidad y respetabilidad de la educación empresarial como disciplina académica y científica, al actuar en muchas ocasiones a través de acuerdos con las universidades.

Tabla 1 - Creación de asociaciones y centros significativos en EE.UU.

\begin{tabular}{|c|c|}
\hline 1956 & $\begin{array}{l}\text { crea el International Council for Small Business (llamado National Council for Small Business } \\
\text { Management Development hasta 1977). }\end{array}$ \\
\hline 1959 & $\begin{array}{l}\text { lanza la SBA Research Initiative (primer esfuerzo público importante para usar académicos en el } \\
\text { estudio de la empresarialidad). }\end{array}$ \\
\hline 1970 & $\begin{array}{l}\text { imer centro de empresarialidad, el Caruth Institute of Owner_Managed Business, establecido en } \\
\text { la Southern Methodist University. }\end{array}$ \\
\hline 1972 & $\begin{array}{l}\text { lanza el programa Small Business Institute por parte de la Small Business Administration en } \\
\text { Texas Tech University. }\end{array}$ \\
\hline 1974 & $\begin{array}{l}\text { forma el Entrepreneurship Interest Group en la Academy of Management bajo la dirección de } \\
\text { Karl Vesper (desde 1987, Entrepreneurship Division). }\end{array}$ \\
\hline 1982 & crea la Association of Private Enterprise Education. \\
\hline 1984 & $\begin{array}{l}\text { ofrece por primera vez el Price_Babson College Fellows Program (programa de formación } \\
\text { pionero para docentes universitarios en empresarialidad). } \\
\text { imera competición de planes de negocio en los campus de Babson College y University of } \\
\text { Texas_Austin (conocido como MOOT CORP). }\end{array}$ \\
\hline 1987 & imera National Business Plan Competition, San Diego State University. \\
\hline 1998 & $\begin{array}{l}\text { uSME, Virtual University for Small and Medium Enterprises en internet (primer programa de } \\
\text { educación empresarial a distancia universitario, en este caso por un consorcio de cuatro facultades). }\end{array}$ \\
\hline
\end{tabular}

En este sentido de la consolidación de la educación empresarial como disciplina, además de la actuación pública indicada, cabe señalar otros dos tipos de acontecimientos. En primer lugar, la creación de asociaciones y centros dedicados a la investigación y divulgación sobre el tema. En segundo lugar, la celebración de reuniones científicas y la aparición de revistas científicas en ese 
ámbito. Respecto al primero de ellos, Katz (2003) apunta en su cronología la creación de algunas de las más significativas asociaciones en este campo, según se señala en la Tabla 1.

Como puede verse, desde muy pronto comienzan a crearse asociaciones científicas para el estudio de la empresarialidad y la pequeña empresa. Aunque su objetivo principal es el estudio del factor empresarial, no cabe duda que la especialización investigadora en un determinado campo favorece el desarrollo de cursos formativos en el mismo. Por ello, si bien es cierto que hasta 1982 no surge una institución exclusivamente dedicada a la educación empresarial, las surgidas anteriormente han contribuido indudablemente al desarrollo de ese tipo de formación en las universidades norteamericanas.

Tabla 2 - Revistas científicas y congresos sobre educación empresarial en EE.UU.

\begin{tabular}{|c|c|}
\hline 1949 & $\begin{array}{l}\text { omienza a publicarse Explorations in entrepreneurial history en Harvard (Primera revista de } \\
\text { investigación centrada en los empresarios. Dejó de publicarse en 1969) }\end{array}$ \\
\hline 1963 & Comienza a publicarse Journal of Small Business Management (JSBM). \\
\hline 1970 & $\begin{array}{l}\text { rimer gran congreso académico organizado por John Komives y Arnold Cooper en Purdue } \\
\text { University, Symposium on technical entrepreneurship. }\end{array}$ \\
\hline 1975 & $\begin{array}{l}\text { elebración del International Symposium of Entrepreneurship and enterprise development, en } \\
\text { Cincinnati }\end{array}$ \\
\hline 1976 & $\begin{array}{l}\text { omienza a publicarse American Journal of Small Business. Desde } 1988 \text { se denomina } \\
\text { Entrepreneurship: Theory \& Practice. }\end{array}$ \\
\hline 1977 & Comienza a publicarse Entrepreneur Magazine (revista de difusión) \\
\hline 1979 & Comienza a publicarse Inc. (Revista de difusión) \\
\hline 1980 & Primera congreso State of the art celebrada en Baylor University \\
\hline 1981 & Primer congreso Babson Entrepreneurship Research Conference \\
\hline 1985 & Comienza a publicarse Journal of Business Venturing \\
\hline 1986 & Comienza a publicarse Advances in the study of entrepreneurship, innovation and economic growth \\
\hline 1988 & Comienza a publicarse Family Business Review \\
\hline 1989 & Comienza a publicarse Small Business Economics \\
\hline 1993 & Comienza a publicarse Advances in entrepreneurship, firm emergence and growth \\
\hline
\end{tabular}

Fuente: Katz, J.A. (2003) y Veciana, J.M. (1999).

Respecto al segundo de los acontecimientos que contribuyen a la consolidación de la educación empresarial como disciplina -la celebración de reuniones científicas-, la Tabla 2 recoge el surgimiento de las más importantes en los Estados Unidos, así como la aparición de algunas de las revistas más importantes especializadas en el área de la empresarialidad. Como puede observarse, algunas de esas publicaciones son bastante antiguas. Este es el caso sobre todo de Journal of Small Business Management y, algo más tarde, la que luego sería Entrepreneurship Theory and Practice.

Por último, el aspecto donde el desarrollo de la educación empresarial está siendo más tardío es sin duda en el de los programas de doctorado. De hecho, Katz (2003) sólo enumera seis 
programas de doctorado específicos sobre empresarialidad, de los cuáles uno es canadiense y dos más europeos. Sin embargo, en esa misma lista se identifican otras veintidós universidades estadounidenses en las que existe un programa de doctorado que si bien no es específicamente sobre empresarialidad, sí que ofrece la posibilidad de especialización en ese área.

Se puede decir, por tanto, que el desarrollo de la disciplina en los Estados Unidos ha sido espectacular. Así, Vesper; McMullan (1988) afirman que hacia el final de los años ochenta, entre un $10 \%$ y un $25 \%$ de todos los que obtienen su título de Máster en Administración de Empresas han elegido al menos un curso sobre empresarialidad. No se trata ya sólo de que la educación empresarial partiera de su práctica inexistencia hace cincuenta años, sino que incluso hace veinte todavía padecía una importante falta de reconocimiento y consolidación a nivel académico y científico. A pesar de todo ello, en la actualidad ya nadie duda de su consolidación ni de su futuro.

\section{Visión de la educación empresarial en Europa}

El desarrollo de la educación empresarial en Europa ha sido mucho más modesto y tardío que en los Estados Unidos. Las primeras experiencias datan de los años setenta. Así, por ejemplo, en el Reino Unido los primeros intentos de definir esta disciplina surgieron hacia 1975, cuando se celebró la primera reunión de la Asociación de Educación sobre Gestión de Pequeñas Empresas (UK Small Business Management Education Association) como grupo informal de académicos interesados en impulsar el desarrollo de las pequeñas empresas (BIRLEY; GIBB, 1984). En el caso de Francia, fueron las Escuelas Superiores de Negocio (Hautes Écoles Commerciales) las que comenzaron a poner en marcha a partir de 1978 ciertas experiencias formativas centradas en la creación de empresas (FAYOLLE, 1999).

No obstante, aunque existe una importante coincidencia a la hora de señalar a Europa entre las áreas donde la educación empresarial está más desarrollada, es muy difícil cuantificar ese nivel de difusión. A diferencia de lo que ocurre en Norteamérica, aquí no existen grandes asociaciones que aglutinen a la mayoría de los investigadores y docentes, y que al mismo tiempo sean reconocidas como referencia en el campo. Por tanto, no hay un gran "punto de encuentro" a partir del cual estudiar la situación. A esto hay que añadir las diferencias nacionales en cuanto a la organización del sistema educativo o el grado de impulso político. Por tanto, el estudio de la situación actual en Europa es muy complicado.

A pesar de ello, son varios los casos de investigadores que han tratado de describir la situación en un país concreto del continente, como Fayolle (1999) u Obrecht (1998) en Francia, Klandt (2000) en Alemania, o Rosa (1992) en el Reino Unido. En cambio, entre los intentos de 
describir la situación global en el continente europeo cabe citar a Carsrud (1991), Dana (1992), o Hisrich; O'Cinneide (1996).

El trabajo de Carsrud (1991) trata de explicar algunas de las diferencias existentes entre los sistemas educativos europeos y norteamericanos. Así, destaca en primer lugar, el hecho de que en Europa esos sistemas educativos son en general bastante más centralizados y existen muchas menos universidades privadas. En consecuencia, los planes de estudio, sistemas de enseñanza, etc., son mucho más difíciles de modificar, pues requieren una decisión a nivel nacional. Por tanto, no es extraño que las grandes universidades europeas hayan sido muy reacias a adoptar la educación empresarial. En cambio, los politécnicos habrían sido más receptivos a esta corriente. Además, según ese autor, la idea de que los profesores universitarios sean al mismo tiempo empresarios en activo o consultores tiende a ser desaprobada en Europa. Del mismo modo, ese sistema centralizado y basado principalmente en universidades públicas ayuda a explicar por qué existen relativamente pocas cátedras dotadas (privadamente) para el estudio y enseñanza de la empresarialidad. Las que existen suelen estar en universidades privadas como INSEAD (Francia), IMEDE (Suiza) o Cranfield School of Management (Reino Unido).

En segundo lugar, Carsrud (1991) señala el relativamente mayor énfasis europeo en el desarrollo económico regional como uno de los elementos fundamentales para el estudio de la empresarialidad. En este sentido, esto podría justificar una mayor tendencia europea hacia el análisis de los aspectos del entorno que dificultan la actividad empresarial, relegando los aspectos personales y motivacionales a un segundo término.

Hisrich; O'Cinneide (1996), por su parte, llevan a cabo un estudio bastante detallado mediante encuestas a centros de educación superior. La Tabla 3 refleja el número de instituciones a las que se envió el cuestionario en cada país, así como las que respondieron al mismo, en orden decreciente. A partir de la primera columna, parece evidente que Reino Unido y Francia son los dos países de los que se tienen más referencias de actividades de educación empresarial, a pesar de la tan diferente tasa de respuesta. En el resto de Europa Occidental existe también un significativo número de instituciones que realizan algún tipo de actividad relacionada con la empresarialidad. 
Tabla 3 - Actividades de investigación y educación empresarial en Europa.

\begin{tabular}{|c|c|c|c|c|c|c|}
\hline \multirow{2}{*}{ País } & \multicolumn{2}{|c|}{ Muestra } & \multicolumn{4}{|c|}{ Tipo de actividad } \\
\hline & Enviadas & Contestadas & Educación & Capacitación & Investigación & Creación \\
\hline Reino Unido & 32 & 25 & 18 & 16 & 20 & 3 \\
\hline Francia & 26 & 9 & 5 & 3 & 4 & - \\
\hline Alemania & 15 & 8 & 3 & 2 & 6 & 1 \\
\hline Holanda & 14 & 5 & 3 & 2 & 4 & - \\
\hline Irlanda & 13 & 5 & 4 & 3 & 3 & 1 \\
\hline Italia & 13 & 2 & 1 & 2 & 2 & - \\
\hline Bélgica & 12 & 9 & 4 & 6 & 4 & 1 \\
\hline Finlandia & 12 & 7 & 4 & 6 & 6 & - \\
\hline Suecia & 11 & 5 & 3 & 1 & 4 & 1 \\
\hline España & 11 & 2 & 1 & - & 1 & 1 \\
\hline Total & 227 & 109 & $\ddot{64}$ & 63 & 69 & 13 \\
\hline
\end{tabular}

Fuente: Elaboración propia con datos de Hisrich, R.D. y O'Cinneide, B., (1996).

El estudio indaga no sólo sobre las actividades de formación en sí, sino también sobre las actividades investigadoras y sobre las de apoyo efectivo a la creación de empresas. Así, la actividad más frecuente es la investigación en el área de la empresarialidad, que se da en casi dos tercios de las respuestas. Sin embargo, según Hisrich O’Cinneide (1996), la mayoría de esa investigación se centra en las pequeñas empresas, y no en la creación de nuevos proyectos.

En lo que se refiere a la formación empresarial, el estudio distingue entre la que se dirige a los propios estudiantes ("Educación") y la que se imparte fuera de currículum para empresarios potenciales o en activo (“Capacitación”). En ambos casos, su grado de difusión es bastante elevado, cercano al $60 \%$. En cuanto a la primera de ellas, existe una gran diversidad. Si bien la mayoría de las universidades simplemente ofrecen un curso optativo dentro de los estudios de grado, algunas de ellas extienden ese curso al nivel de postgrado. Otras ofrecen entre dos y cuatro cursos optativos, tanto en los estudios de grado como en masters. Respecto a la "capacitación", en cambio, la variedad es mucho menor. El objetivo principal que se persigue es el de proporcionar a los participantes los conocimientos y procedimientos que les permitan la efectiva puesta en marcha de su empresa, reduciendo la probabilidad de que el nuevo proyecto fracase. Sin embargo, también existen proyectos que se dirigen a otros colectivos como pequeños empresarios, empresas de sectores y/o tamaños determinados, o científicos e ingenieros. Por último, algunos de estos programas obtienen financiación especial de organismos públicos como los ministerios de trabajo.

Los programas para la "creación" efectiva de empresas son la actividad menos frecuente, pues sólo un $12 \%$ de las instituciones las realiza. En todo caso, la forma más habitual de llevarla a cabo consiste en el asesoramiento a individuos ajenos a la universidad. Este servicio se presta a 
veces directamente por la misma, aunque otras veces se hace a través de organismos especiales creados para ello.

En términos generales, en Europa existiría una tendencia a concentrarse en la formación para la gestión de pequeñas empresas, no prestando suficiente atención a los aspectos relacionados con la creación de nuevos proyectos (DANA, 1992; HISRICH; O’CINNEIDE, 1994). Además, el retraso europeo sería especialmente notable en los estudios de postgrado (CARSRUD, 1991; DANA, 1992).

En cuanto a la infraestructura para el estudio de la empresarialidad y, a partir de ahí, para la educación empresarial. Casrud (1991) cita cuatro tipos de elementos de apoyo: centros de investigación; asociaciones científicas; encuentros / congresos; y publicaciones. Respecto a los primeros, ese autor enumera sin ánimo de ser exhaustivo 28 de estos centros. Algunos son ampliamente conocidos, como el Small Business Centre de la Durham University Business School (DUBS), el Swiss Research Institute of Small Business and Entrepreneurship de la Universität St. Gallen, o EIM en Holanda.

Por su parte, Landström et al. (1997) identificaron más de cincuenta cátedras dotadas en universidades y centros de investigación europeos. De nuevo, el Reino Unido con más de una docena y Francia con once destacan sobre los demás. En el resto de países europeos el número de cátedras está entre cero y tres, con las excepciones de Suecia (cuatro) y Finlandia (once). Este último caso es especialmente sorprendente si atendemos a autores como Erkkilä (2000), que afirman que la educación empresarial era prácticamente inexistente en Finlandia antes de 1990, aunque también especifica que ha recibido un gran impulso público desde entonces.

En lo que se refiere a asociaciones científicas, a continuación citamos los más importantes del continente según autores como Carsrud (1991) o Landström et al. (1997). La mayoría de ellas organiza su propio congreso anual:

- European Foundation for Entrepreneurial Research (EFER).

- Entrepreneurship, Innovation and Small Business Chapter (EISB), dentro de la European Foundation for Management Development (EFMD).

- European Council of Small Business (ECSB)

Además, también habría que citar específicamente los congresos Rencontres de St. Gall (Universität St. Gallen), e IntEnt Internationalizing Entrepreneurship Education and Training Conference (sede rotatoria, pero organizado por la European Business School de Alemania).

Por último, en lo referente a las revistas científicas, Carsrud (1991) identifica cuatro publicaciones europeas en el área de la empresarialidad: Entrepreneurship and Regional Development; International Small Business Journal; Small Enterprise Development; y Piccola Impresa. Por su parte, Landström et al. (1997) mencionan otras como por ejemplo: Internationales 
Gewerbearchiv, Small Business Economics ${ }^{1}$, International Journal of Entrepreneurial Behaviour and Research. A éstas habría que añadir, según Liñán (2004), Journal of European Industrial Training.

De esta forma, la percepción europea sobre los objetivos de la educación empresarial se aproxima mucho a lo que Curran; Stanworth (1989) llaman "sensibilización hacia la actividad empresarial", ya que perseguiría desarrollar actitudes y comportamientos más dinámicos, pero sin vincularlos directamente con la creación de empresas (GIBB, 1993; ERKKILÄ, 2000). A este fin principal se orientaría la educación empresarial dentro del sistema educativo. Así, aunque en determinados niveles del mismo podrían desarrollarse iniciativas encaminadas a la "creación de empresas y autoempleo", esto sería especialmente factible en la formación profesional reglada, y en los niveles universitarios. No obstante, incluso en estos casos concretos, la falta de experiencia profesional de los alumnos constituiría un importante obstáculo para que la formación recibida llevara a los participantes a la efectiva creación de una empresa.

Otro aspecto que ha resultado muy significativo en el desarrollo de la educación empresarial en Europa ha sido la promoción de la cultura empresarial. Para Erkkilä (2000), este énfasis pretende lograr construir una sociedad más empresarial. De esta forma, el objetivo sería que incluso aquellos estudiantes que no van a ser empresarios, lleguen a convertirse en individuos emprendedores, y también tengan una opinión más favorable sobre la actividad empresarial.

En este sentido, la definición propuesta por el grupo de trabajo de la Comisión Europea (2002) sobre educación empresarial se encuentra, como cabría esperar, dentro de esa línea, aunque reconoce explícitamente la existencia de esos dos elementos componentes de la educación empresarial: el más amplio referido a la educación en actitudes y capacidades empresariales; y el más específico referido a la formación para la creación de empresas.

En relación concretamente con el sistema educativo reglado, puesto que lo que se persigue es la promoción por parte de los gobiernos de los Estados Miembros el establecimiento de una sociedad más emprendedora, la Comisión Europea (2002: p. 16) define la educación empresarial como aquellas actividades de «enseñanza y aprendizaje del espíritu empresarial [que] incluyen el desarrollo de conocimientos, capacidades, actitudes y cualidades personales adecuadas a la edad y el desarrollo de los escolares o estudiantes».

En la educación primaria, fomentaría en los escolares cualidades personales como la creatividad, el espíritu de iniciativa y la independencia, que contribuyen al desarrollo de una actitud empresarial y que les resultarán útiles en la vida y en cualquier actividad profesional:

Esta revista también la hemos recogido en el apartado referido a los Estados Unidos. Esta duplicación se debe a que los editores de la revista (Z.J.Acs y D.B.Audretsch) trabajan desde los Estados Unidos, pero tanto el editor asociado (A.R. Thurik) como también la empresa editora tienen su sede en Holanda. 
- En la educación secundaria, el desarrollo de esas cualidades personales sigue siendo pertinente. Además, incluiría la concienciación de los estudiantes acerca del empleo por cuenta propia como posible opción profesional, y también formación específica sobre cómo crear una empresa.

- En la educación superior, aportaría a los estudiantes una formación específica sobre cómo poner en marcha y dirigir una empresa. También fomentaría y apoyaría las ideas empresariales, para que los buenos proyectos se puedan poner en práctica y acaben llegando al mercado.

Se trata, como vemos, de una concepción que parte de esa visión más amplia en los niveles inferiores del sistema educativo, pero que va evolucionando en los niveles superiores hacia los aspectos más concretos de la creación de empresas. Así, en la educación primaria y secundaria se fomentarían todo el conjunto de cualidades de los individuos que están relacionadas de un modo más o menos general con la iniciativa empresarial o empresarialidad. Por su parte, sería en los niveles superiores del sistema educativo (secundaria y, sobre todo, universitaria) donde la educación empresarial debe tratar de relacionar y canalizar estas cualidades de forma mucho más específica hacia la creación efectiva de empresas y el autoempleo (COMISIÓN EUROPEA, 2002).

\section{Conclusiones}

El agente empresarial ha adquirido una mayor presencia en las economías de los distintos países desde la crisis de los años setenta e, igualmente, también ha recibido una mayor atención en el terreno académico, así como por parte de los decisores políticos. Todo este proceso ha venido acompañado de un rápido desarrollo de la educación empresarial, especialmente en los Estados Unidos y en Europa. Sin embargo, han existido diferencias muy significativas en la forma en la que se ha producido esa evolución en cada una de esas zonas.

En este trabajo, hemos adoptado la definición de educación empresarial propuesta por Liñán (2004), a partir de la cual hemos clasificado los distintos tipos de educación empresarial que pueden existir. Así, la educación para la calidad empresarial constituye la variante más ambiciosa posible, aunque también la más compleja. Existen pocos ejemplos de esta modalidad formativa en la realidad. Por el contrario, la formación para empresarios en activo sería la más limitada, y sólo adquiriría cierto sentido como complemento de las otras modalidades. En una situación intermedia, la sensibilización hacia la actividad empresarial y la educación para la creación de empresas son las variantes más habituales. Además, entre estas dos últimas categorías es donde se centran las diferencias entre los enfoques estadounidense y europeo.

La educación empresarial se ha desarrollado en primer lugar en los Estados Unidos, acelerando su expansión durante los años setenta y ochenta. Por lo tanto, en la actualidad se haya claramente consolidada. Su enfoque se ha centrado desde el principio en la formación para la 
creación de empresas, sin prestar apenas atención a las otras tres variantes posibles. No obstante, durante los años ochenta y noventa se han comenzado a desarrollar algunas iniciativas de sensibilización dentro del sistema educativo.

Por su parte, el desarrollo seguido en Europa ha sido mucho más tardío. Tan sólo en el Reino Unido y, en menor medida, Francia se pusieron en marcha algunas iniciativas durante los años setenta. En el primero de esos países la evolución fue algo más rápida, produciéndose una importante expansión durante los años ochenta. En el resto del continente, en cambio, no ha sido hasta los años noventa cuando se ha producido la verdadera difusión de la educación empresarial. En particular, la Comisión Europea ha contribuido significativamente desde su Dirección General de Empresa mediante la organización de reuniones, la identificación y divulgación de las mejores prácticas y, en definitiva, mediante una tarea de coordinación muy relevante.

En Europa, partiendo de la experiencia británica, la preocupación principal ha sido lograr establecer una sociedad más empresarial como meta general, y el desarrollo de personalidades emprendedoras o innovadoras por parte de los participantes en ese tipo de formación. Por lo tanto, se trata de una actividad claramente vinculada con la sensibilización hacia la actividad empresarial.

La justificación de esta diferencia de enfoque podría seguramente encontrarse en las disparidades socioculturales entre ambas áreas. En efecto, la sociedad estadounidense es mucho más individualista y también posee un mercado laboral mucho más flexible. Por ello, no sólo no existe ningún sesgo contrario al desempeño de la actividad empresarial, sino que es más bien al contrario. De esta forma, existe una oferta suficientemente alta de individuos con inclinación empresarial. En consecuencia, la formación más adecuada es la que se centra en los pasos y técnicas concretos para la creación de la empresa.

Por el contrario, en Europa, el nivel de protección social es mucho más elevado, y se tiende a otorgar un papel mucho más destacado a las distintas instituciones y organizaciones (Estado, sindicatos, asociaciones, consumidores, etc.). El desempeño de la actividad empresarial no es contemplado de forma tan favorable. En la práctica, una parte muy significativa de la población ni siquiera contempla esa opción de salida profesional. En esta situación, la sensibilización parece la acción más adecuada, por lo que cabe afirmar que la evolución seguida ha sido la más lógica.

No obstante, la tarea por recorrer si se quiere convertir a Europa en la sociedad más dinámica del mundo -como afirma la Comisión Europea (2003)- es bastante ardua. Es importante tener en cuenta que la educación empresarial por si sola no puede transformar los valores de una sociedad. Sin embargo, para provocar una modificación generalizada de la consideración de la opción empresarial se requiere una actuación decidida a lo largo de todo el sistema educativo. Hasta ahora, a pesar de las manifestaciones de buena voluntad realizadas, sólo algunos países concretos como Finlandia parecen haber avanzado significativamente en esa dirección. En cualquier caso, los 
resultados sólo se pondrían de manifiesto a largo plazo, a medida que los estudiantes vayan completando su paso por el sistema educativo.

Por último, queremos realizar una llamada de atención sobre los posibles peligros o consecuencias negativas que tendría una estrategia de difusión generalizada de actitudes individualistas y competitivas entre los europeos. Es muy posible que esas medidas llevasen a una mayor tasa de creación de empresas. Sin embargo, podría producirse simultáneamente una reducción en los niveles de solidaridad y conciencia social de los ciudadanos. En este sentido, si bien cabría argumentar la posibilidad de desarrollar una conciencia empresarial con un elevado componente social, no está claro que esto sea factible en la práctica.

\section{ABSTRACT}

Business education acquired a great development in the last decades. This high point was simultaneous to the development of the business community, which receives a greater importance in the processes of economy structural changes. More and more it is recognized the necessity of more and better businessmen to keep the dynamism of the economies. In this sense, business education seems to be one of the most adjusted strategies for business promotion. Undoubtedly, as it happens for a proper definition of businessman there is no unanimity for a concept and objectives of business education. Thus, a business formation program, which searches to find businessmen, can be considered an initiative of business education. Nevertheless, in different levels to apply this vision in one or another direction. Particularly, there are differences in the vision that predominates in the United States and Europe about the business activity in a general way and, in this case about business education. Thus, the main objective of this work is to analyze the differences of business education in each side of the Atlantic and their consequences. These differences allowed analyzing the occurred differences in business education in the two searched regions, as well as their distinct objectives. In the case of Europe, business education spread out more recently, and its objectives are ampler than those that persist in the United States.

Key words: Business education, businessmen, differences.

\section{Referencias}

ACS, Z. J.; AUDRETSCH, D. B. Small firms in the 1990s. In: Acs, Z. J.; Audretsch, D. B. (Eds.). The economics of small firms: a European challenge. Dortrecht : Kluwer Academic Publishers, 1990.

ACS, Z. J.; AUDRETSCH, D. B.; CARLSSON, B. Flexibility, plant size and industrial restructuring. In: ACS, Z. J.; AUDRETSCH, D. B. (Eds.). The economics of small firms: a European challenge. Dortrecht : Kluwer Academic Publishers, 1990.

AYDALOT, P. The role of small and medium-sized enterprises in regional development: conclusions drawn from recent surveys. In: GIAOUTZI, M.; NIJKAMP, P.; STOREY, D. J. (Eds). Small and medium-sized enterprises and regional development. Londres : Routledge. 1988.

BIRCH, D. L. The job generation process, MIT Program on Neighborhood and Regional Change. Cambridge (Mass.). 1979.

BIRLEY, S.; GIBB, A. A. Teaching small business management in the UK. Part I. Journal of European Industrial Training, v. 8, n. 4, p. 17-24, 1984. 
BLACKMAN, R.; THOMPSON, J. H. The 1986 White House Conference on small business. Journal of Small Business Management, v. 25, n. 1, p. 3-10, 1987.

BROCK, W. A.; EVANS, D. S. Small business economics. Small Business Economics, v. 1, n. 1, p. 7-20, 1989.

BROCKHAUS, R. H. Entrepreneurship education: a research agenda. In: INTERNATIONALIZING ENTREPRENEURSHIP EDUCATION AND TRAINING, 1992, Dortmund. Anais... Dortmund, 1992.

CARREE, M. A.; THURIK, A. R. Small firms and economic growth in Europe. Atlantic Economic Journal, v. 26, n. 2, p. 137-146, jun. 1998.

CARSRUD, A. L. Entrepreneurship and enterprise formation: a brief perspective of the infraestructure in Europe. Entrepreneurship Theory and Practice, v. 15, n. 3, p. 69-75, 1991.

COHEN, W. M.; KLEPPER, S. The tradeoff between firm size and diversity in the pursuit of technological progress. Small Business Economics, v. 4, n. 1, p. 1-14, 1992.

COHEN, W. M.; KLEPPER, S. A reprise of size and R \& D. The Economic Journal, v. 106, p. 925-951, jul. 1996.

COMISIÓN EUROPEA. Enterprises in Europe. $5^{\text {th }}$ ed. Luxemburgo : Oficina de Publicaciones de la Comisión Europea, 1998.

COMISIÓN EUROPEA. Action Plan to Promote Entrepreneurship and Competitiveness. Luxemburgo : Oficina de Publicaciones de la Comisión Europea, 1999.

COMISIÓN EUROPEA. Informe final del grupo de expertos. Proyecto del «procedimiento best» sobre Educación y formación en el espíritu empresarial. Bruselas : Dirección General de Empresa, 2002.

COMISIÓN EUROPEA Libro verde. El espíritu empresarial en Europa. Bruselas : Dirección General de Empresa, 2003.

CURRAN, J.; STANWORTH, J. Education and training for enterprise: some problems o classification, evaluation, policy and research. Internacional Small Business Journal, v. 7, n. 2, p. 11-22, 1989.

DANA, L. P. Entrepreneurial education in Europe. Journal of Education for Business, v. 68, n. 2, p. 74-78, 1992.

ERKKILÄ, K. Entrepreneurial Education. Mapping the debates in the United States, the United Kingdom and Finland. New York : Garland Publishing, 2000.

FAYOLLE, A. L'enseignement de l'entrepreneuriat dans les universités françaises: analyse de l'existant et propositions pour en faciliter le développement. París : Ministerio de Educación Nacional, de Investigación y de Tecnología, 1999.

FISHER, M. M. Business formation and regional development: some major issues. In: GIAOUTZI, M., NIJKAMP, P.; STOREY, D. J. (Eds.). Small and medium-sized enterprises and regional development. London : Routledge, 1988.

GARAVAN, T. N.; O'CINNEIDE, B. Entrepreneurship education and training programmes: a review and evaluation. Journal of European Industrial Training, v. 18, n. 8, p. 3-12, 1994.

GIBB, A. A. Entrepreneurship and intrapreneurship - Exploring the differences. In: DONKELS, R.; MIETTINEN, A. (Eds.). New findings and perspectivas in entrepreneurship. Aldershot : Avebury Gower Publishing, 1990.

GIBB, A. A. The enterprise culture and education. Understanding enterprise education and its links with small business entrepreneurship and wider educational goals. International Small Business Journal, v. 3, n. $11,1993$.

HISRICH, R. D.; O'CINNEIDE, B. Entrepreneurial activities in Europe-oriented institutions. Journal of Managerial Psychology, v. 11, n. 2, p. 45-64, 1996.

KATZ, J. A. The chronology and intellectual trajectory of American entrepreneurship education: 1876-1999. Journal of Business Venturing, v. 18, p. 283-300, 2003. 
KENT, C. A. Entrepreneurship education at the collegiate level: a synopsis and evaluation. In: KENT, C. A. (Ed.). Entrepreneurship education: current developments, future directions. Westport : Quorum Books, 1990.

KIRCHHOFF, B. A. Creative destruction among industrial firms in the United States. In: ACS, Z. J.; AUDRETSCH, D. B. (Eds.). The economics of small firms: a European challenge. Dordrecht : Kluwer Academic Publishers, 1990.

KLANDT, H. Entrepreneurship climate in Germany and entrepreneurship education at the EBS. In: INTERNATIONALIZAING ENTREPRENEURSHIP EDUCATION AND TRAINING CONFERENCE, 2000, Tampere. Anais... Tampere, 2000.

LANDSTRÖM, H.; FRANK, H.; VECIANA, J. M. Entrepreneurship and small business researh in Europe: an ECSB survey. Aldershot : Ashgate, 1997.

LIÑÁN, F. Hacia una nueva política de fomento empresarial: al apoyo a la esfera impulsora del empresario. 2001. Dissertácion (Mestrado em Economía Aplicada) - Departamento Economía Aplicada I, Universidad de Sevilla, Sevilla, 2001.

LIÑÁN, F. Educación empresarial y modelo de intenciones. Formación para un empresariado de calidad. 2004. Tesis (Doctorado en Economía Aplicada) - Departamento Economía Aplicada I, Universidad de Sevilla, Sevilla, 2004.

LOUCKS, K. E. Training entrepreneurs for small business creation. Ginebra : International Labour Office, 1988.

LOVEMAN, G.; SENGERBERGER, W. The re-emergence of small scale production: an international comparison. Small Business Economics, v. 3, n. 1, p. 1-37, 1991.

MAILLAT, D. The role of innovative small and medium-sized enterprises and the revival of traditionally industrial regions. In: GIAOUTZI, M., NIJKAMP, P.; STOREY, D. J. (Eds). Small and medium-sized enterprises and regional development. Londres : Routledge, 1988.

McINTYRE, J. R.; ROCHE, M. University education for entrepreneurs in the United States: a critical and retrospective analysis of trends in the 1990s. Center for International Business Education and Research 1999-2000 Working Paper Series, num. 99/00-021. Atlanta : Georgia Institute of Technology, 1999.

McMULLAN, W. E.; GILLIN, L. M. Developing technological start-up entrepreneurs: a case study of a graduate entrepreneurship programme at Swinburne University. Technovation, v. 18, n. 4, p. 275-286, 1998.

McMULLAN, W. E.; LONG, W. A. Entrepreneurship education in the nineties. Journal of Business Venturing, v. 2 , n. 3, p. 261-275, 1987.

NICKEL, S. J. Competition and corporate performance. Journal of Political Economy, v. 104, n. 4, p. 724-746, 1996.

OBRECHET, J. J. Entrepreneurship education and training in France a new challenge to the universities. In: INTERNATIONALIZING ENTREPRENEURSHIP EDUCATION AND TRAINING, 1998, Oestrich-Winkel. Anais... Oestrich-Winkel, 1998.

OCDE. Estimular el espíritu empresarial. Paris : Organización para la Cooperación y el Desarrollo Económico, 1999.

PIORE, M. J.; SABEL, C. La segunda ruptura industrial. Madrid : Alianza Universidad, 1990.

PLASCHKA, G. R.; WELSCH, H. P. Emerging structures in entrepreneurship education: curricular designs and strategies. Entrepreneurship Theory and Practice, v. 14, n. 3, p. 55-71, 1990.

RONDINELLI, D. A.; KASARDA, J. D. Foreign trade potential, small enterprise development and job creation in developing countries. Small Business Economics, v. 4, p. 253-265, 1992.

ROSA, P. Entrepreneurial training in the UK: past confusion and future promise. In: KLANDT, H.; MULLERBOLING, D. (Eds.). Internationalizing Entrepreneurship Education and Training. Koln Dortmund : ForderKreis Grundungs-Forschung, 1992.

SANTOS, F. J. La calidad del empresario sevillano. Sevilla : Biblioteca de Socioeconomía Sevillana, Sevilla Siglo XXI, 2001. 
SBA. Building the Foundation for the new century. [Report on the implementation of the White House Conference]. Washington, 2000.

SBA. 49 years of service to America's small business: the U.S. Small Business Administration, $1953-2002$. Washington : Small Business Administration, 2002.

SCHUMPETER, J. A. Teoría del desenvolvimiento económico. México : Fondo de Cultura Económica, 1967.

SCHWALBACH, J. Small business dynamics in Europe. Small Business Economics, v. 6, n. 1, p. 21-25, 1994.

SEXTON, D. L.; BOWMAN, N. B. Entrepreneurship education: suggestions for increasing effectiveness. Journal of Small Business Management, v. 22, n. 2, p. 18-25, 1984.

SOLOMON, G. T.; WEAVER, K. M.; FERNALD, L. W. A historical examination of small business management and entrepreneurship pedagogy. Simulation and Gaming, v. 25, n. 3, p. 338-352, 1994.

STOREY, D. J. Entrepreneurship and the new firm. Beckenham, Kent : Croom Helm, 1982.

STOREY, D. J. The role of small an medium-sized enterprises in European job creation. In: GIAOUTZI, M.; NIJKAMP, P.; STOREY, D. J. (Eds.). Small and medium-sized enterprises and regional development. London : Routledge, 1988.

TIMMONS, J. A. New venture creation: entrepreneurship for the $21^{\text {st }}$ century. 5. ed. Singapore : Irwin-McGraw-Hill, 1999.

VECIANA, J. M. Creación de empresas como programa de investigación científica. Revista Europea de Dirección y Economía de la Empresa, v. 8, n. 3, p. 11-36, 1999.

VESPER, K. H. Research on education for entrepreneurship. In: KENT, C. A.; SEXTON D. L.; VESPER K. H. (Eds.). Encyclopedia of entrepreneurship. Englewood Cliffs : Prentice Hall. 1982.

VESPER. K. H.; GARTNER, W. B. Measuring progress in entrepreneurship education. Journal of Business Venturing, v. 12, p. 403-421, 1997.

VESPER, K. H.; McMULLAN, W. E. Entrepreneurship: today courses tomorrow degrees? Entrepreneurship Theory and Practice, v. 3, n. 1, p. 7- 13, 1988.

WEINRAUCH, J. D. Educating the entrepreneur: understanding adult learning behavior. Journal of Small Business Management, v. 22, n. 2, p. 32-37, 1984.

WENNEKERS, S.; THURIK, R. Linking entrepreneurship and economic growth. Small Business Economics, v. 13, p. 27-55, 1999. 